\title{
Chosen problems with acquiring multispectral imagery data using the MiniMCA camera
}

\author{
Rafal Dabrowski, Agata Orych \\ ${ }^{a}$ Department of Remote Sensing and Photogrammetry, \\ Institute of Geodesy, Faculty of Civil Engineering and Geodesy, \\ Military University of Technology ul. Gen. Sylvester Kaliski 2 00-908 Warsaw 49, Poland
}

\begin{abstract}
In recent years there had been a visible increase in the availability of light - "amateur" UAV's, which can be adapted for remote sensing applications, e.g. research concerning water pollution. One of the many methods used to detect pollutants in water is a method based on spectral reflectance coefficients.

Spectral reflectance coefficients can be obtained from imagery acquired in different spectra due to their close correlation to the pixel value. The miniMCA6-channel camera had been specially designed for UAV applications. It enables the acquisition of multispectral imagery data in varied ranges of the electromagnetic spectrum, the bandwidths of which are determined by the applied interference filters. One of its biggest drawbacks is the inability to set exposure parameters for each channel separately.

Depending on the transmission coefficient of the interference filters used for each channel, the exposure time for each channel should be different. This can be only adjusted using a numerical value linking the exposure time of the master channel with the exposure time of the slave channel depending on the used interference filter. Therefore the value of a single pixel in the individual spectral channels are often burdened with errors.

This phenomenon is most noticeable when acquiring imagery of spectral reflectance standards. Pixels representing the white reference standards on an image acquired by a slave channel are usually overexposed, that is not the case in the master channel, which has a negative effect on the possibility of acquiring reliable spectral reflectance coefficients of investigated samples.

The article contains a description and the results of an experiment to determine the optimal exposure time for the miniMCA camera (as a function of light intensity and distance to the sample), to ensure that the digital number of the pixels representing the white reference standards is always a chosen stable value.
\end{abstract}

Keywords: Multispectral imagery data; data processing; miniMCA; UAV.

\section{Introduction}

In the past few years there has been a significant increase in the availability of imagery data, especially that obtained from relatively "cheap" sensors placed on unmanned aerial vehicles [1], [5]. These sensors are more and more often characterized by an image recording capacity in selected narrow ranges of the EM spectrum, which until recently was reserved exclusively for expensive imaging systems. Imagery data acquired from UAV sensors are the main source of data in typical photogrammetry projects but also in other tasks e.g. precision agriculture, detection of water pollution or asks decision support [6], [3]. An example of such relatively "low cost" sensor is the TETRACam miniMCA camera [4], [2].

The miniMCA 6-channel camera had been specially designed for UAV applications. [1] It enables the acquisition of multispectral imagery data in varied ranges of the electromagnetic spectrum, the bandwidths of which are determined by the applied interference filters. One of its biggest drawbacks is the inability to set exposure parameters for each channel separately.

Depending on the transmission coefficient of the interference filters used for each channel, the exposure time for each channel, should be different. This can be only adjusted using a numerical value linking the exposure time of the master channel with the exposure time of the slave channel depending on the used interference filter. Therefore the value of a single pixel in the individual spectral channels are often burdened with errors.

Corresponding author: Rafal Dabrowski. E-mail address: dabrowski@wat.edu.pl

http://dx.doi.org/10.3846/enviro.2014.010

(C) 2014 The Authors. Published by VGTU Press. This is an open-access article distributed under the terms of the Creative Commons Attribution License, which permits unrestricted use, distribution, and reproduction in any medium, provided the original author and source are credited. 
This phenomenon is most noticeable when acquiring imagery of spectral reflectance standards. Pixels representing the white reference standards on an image acquired by a slave channel are usually overexposed, that is not the case in the master channel, which has a negative effect on the possibility of acquiring reliable spectral reflectance coefficients of investigated samples.

The article contains a description and the results of an experiment to determine the optimal exposure time for the miniMCA camera (as a function of light intensity and distance to the sample), to ensure that the digital number of the pixels representing the white reference standards is always a chosen stable value.

As a result a nomogram was created (for each channel) for defining the optimal exposure time for a given luminance (lux) for obtaining a set Digital Number (DN) value of a white reference panel.

This will lead to a possibility of registering reliable spectral data of studied samples without the need of having a white reference panel present within the scene.

\section{MiniMCA camera}

The compact multispectral miniMCA camera has been specially designed to be mounted on board an unmanned aerial vehicle. The camera weighs 700 grams and its dimensions are $114 \times 78 \times 80 \mathrm{~mm}$. The tested camera was equipped with six separate CMOS sensors effective spectral range of data acquisition of 400-950 nm. The part of the EM spectrum which will be imaged is determined by the applied interference filters. The camera uses standard interference filters with a diameter of $25 \mathrm{~mm}$. It is possible to replace the cameras filters. The tested camera uses filters with values $\lambda \max =550,490,610,660$, $720,850 \mathrm{~nm}$.

The matrix resolution of each sensor is $1280 \times 1024$ with a pixel size of 5.2 microns. The radiometric resolution is 10 -bit. The camera uses a standard USB interface for communication and control of operations. An additional and very important advantage of the camera MCA 6 is its ability to collect data directly to a memory card. For registering data from each sensor a data card with a capacity of 2 GB can be used, which gives an effective memory of up to 12 GB. The camera also allows you to connect an external GPS receiver [4].

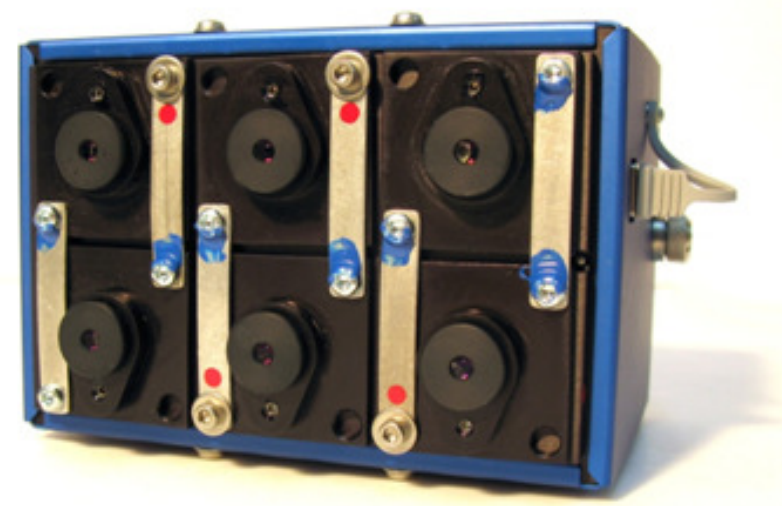

Fig. 1. MiniMCA multispectral camera

You can store data in three file formats: RAW8, RAW10 and DCM 10. The advantages and disadvantages are shown in Table 1. The fastest image data acquisition cycle saves data in a RAW8 format. The cycles speed depends on the memory card and is about 1 image per second. When imagery with a higher radiometric resolution is needed, the RAW10 format should be used. This format is "slower" than the data stored in RAW 8. One image saved as RAW10 takes up about 6MB whilst a RAW8 file - about 3 MB. The DCM format (compressed data format) takes up about 3 MB but it takes longer to write the same data. The advantages and disadvantages of these formats is shown in Table 1.

Table 1. Advantages and disadvantages of camera file formats

\begin{tabular}{|l|l|l|}
\hline File format & Advantages & Disadvantages \\
\hline RAW8 & Fastest cycle time & $\begin{array}{l}\text { Less dynamic range, no } \\
\text { embedded previews }\end{array}$ \\
\hline RAW10 & $\begin{array}{l}\text { Fastest cycle time with } \\
\text { full dynamic range }\end{array}$ & $\begin{array}{l}\text { Big files, no embedded } \\
\text { previews }\end{array}$ \\
\hline DCIM10 & $\begin{array}{l}\text { Smallest file size with } \\
\text { full dynamic range }\end{array}$ & $\begin{array}{l}\text { Longest time between } \\
\text { pictures (up to 5 seconds) }\end{array}$ \\
\hline
\end{tabular}




\section{Experiment description}

The aim of this experiment to determine the optimal exposure time for the miniMCA camera (as a function of light intensity and distance to the sample), to ensure that the pixel digital number representing the white reference standard is always a chosen constant value.

Research was conducted in laboratory conditions. The measurement set-up consisted of a miniMCA camera, a screen on which imagery was displayed in real-time, a white reference standard (Zenith Lite SG3151), a luminance meter and a light source (ASD Pro Lamp). Measurements were taken at five difference distances away from the reference panel: $1.8 \mathrm{~m}$; $3.6 \mathrm{~m} ; 5.4 \mathrm{~m}, 7.2 \mathrm{~m} ; 9 \mathrm{~m}$. Figure 2 is a diagram showing the experiment set-up.

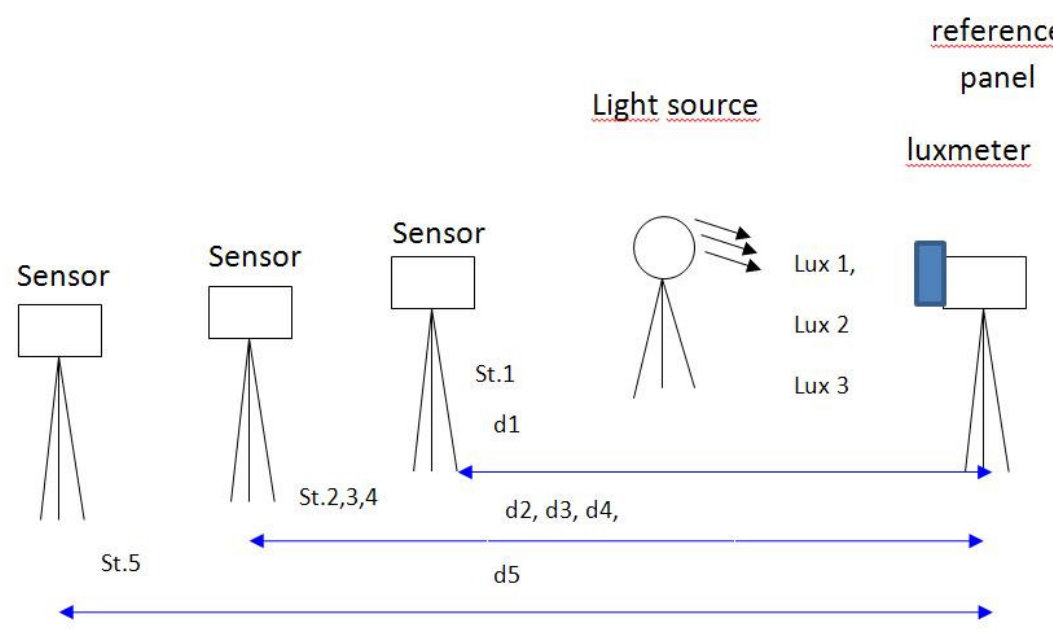

Fig. 2. Diagram of the experiment set-up in laboratory conditions

Three lamps were used to illuminate the reference panel making it possible to obtain three unique but repeatable values of illumination on the scene. Imagery was acquired at three difference illuminations later called Lux1, Lux2 and Lux3. Fluctuations between illuminations on subsequent distances were no greater than \pm 10 lux. Table 2 presents the amount of light incident on the reference panel at each distance:

Table 2. Illuminance at each distance

\begin{tabular}{|l|l|l|l|}
\hline $\begin{array}{c}\text { Distance / } \\
\text { Luminance level }\end{array}$ & Lux 1[1x] & Lux 2 [lx] & Lux3 [lx] \\
\hline $1(1.8 \mathrm{~m})$ & 3070 & 2420 & 1640 \\
\hline $2(3.6 \mathrm{~m})$ & 3070 & 2410 & 1630 \\
\hline $3(5.4 \mathrm{~m})$ & 3070 & 2430 & 1640 \\
\hline $4(7.2 \mathrm{~m})$ & 3070 & 2420 & 1640 \\
\hline $5(9.0 \mathrm{~m})$ & 3070 & 2420 & 1630 \\
\hline
\end{tabular}

At each distance, imagery data was acquired with exposure times from $0,5 \mathrm{~ms}$ do $25 \mathrm{~ms}$ with $5 \mathrm{~ms}$ intervals. During his experiment 5116 images had been registered.

Imagery data was recorded in the RAW10 format. Acquired images were then opened in ERDAS software in order to extract DN information for pixels on the white reference panel. As assumption was made that the DN value of an object on the image is directly correlated with its spectral reflectance coefficient. DN measurements on each of the 6 channels (Master, Slave1, Slave2, Slave3, Slave4, Slave5) were conducted in 5 points distributed evenly on the reference panel. The image coordinates of these points are shown in Table 3.

Table 3. - image coordinates of measurement points

\begin{tabular}{|l|l|l|l|l|l|l|l|l|l|l|}
\hline & $\mathrm{x}$ & $\mathrm{y}$ & $\mathrm{x}$ & $\mathrm{y}$ & $\mathrm{x}$ & $\mathrm{Y}$ & $\mathrm{x}$ & $\mathrm{Y}$ & $\mathrm{X}$ & $\mathrm{Y}$ \\
\cline { 2 - 13 } & $1.8 \mathrm{~m}$ & \multicolumn{3}{|l|}{$3.6 \mathrm{~m}$} & \multicolumn{2}{|l|}{$5.4 \mathrm{~m}$} & \multicolumn{2}{l|}{$7.2 \mathrm{~m}$} & \multicolumn{2}{l|}{$9.0 \mathrm{~m}$} \\
\hline Master & 681 & 645 & 631 & 593 & 583 & 626 & 613 & 555 & 619 & 563 \\
\hline Slave1 & 765 & 641 & 674 & 591 & 612 & 625 & 634 & 554 & 636 & 562 \\
\hline Slave2 & 761 & 548 & 672 & 543 & 610 & 594 & 633 & 530 & 635 & 543 \\
\hline Slave3 & 687 & 543 & 634 & 541 & 585 & 592 & 614 & 529 & 620 & 542 \\
\hline Slave4 & 729 & 592 & 655 & 566 & 599 & 609 & 625 & 541 & 629 & 552 \\
\hline Slave5 & 681 & 645 & 631 & 593 & 583 & 626 & 613 & 555 & 619 & 563 \\
\hline
\end{tabular}


As a result, we obtained DN values for all measurement points as well as standard deviation, minimum and maximum DN values. Further results are based on average values from these $5 \mathrm{DN}$ values.

\section{Analysis of results}

Firstly, the acquired data was analyzed in terms of the impact of the exposure time on the digital number of the pixel at different illuminations (Lux1, Lux2 and Lux3). An example of this dependence for the first measuring distance had been shown in Figure 3. Each colour represents a different illumination of the scene.

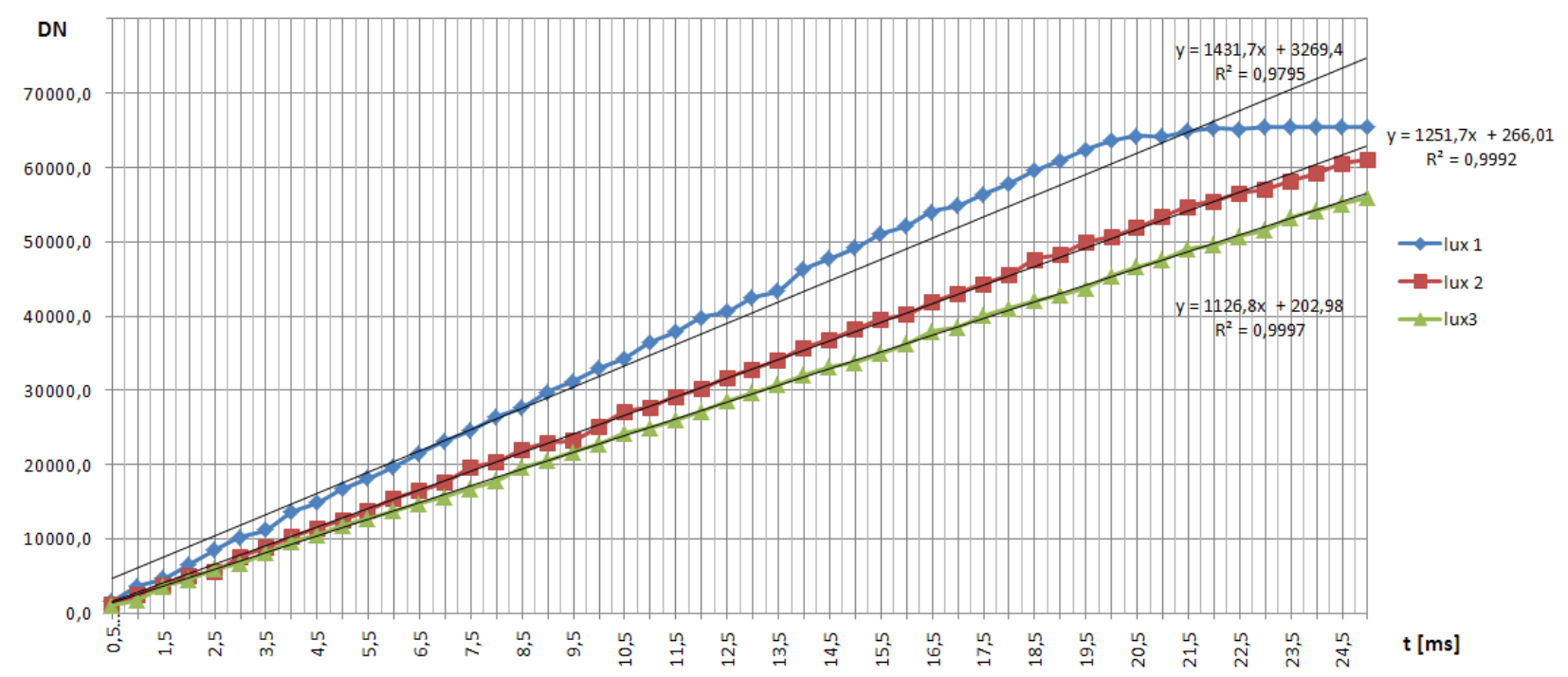

Fig. 3. The dependence between the exposure time and the DN value for three different illuminations - Distance 1

By analyzing the acquired data it is visible that for every distance, the dependence between the exposure time and DN value can be described by a linear function. The slope of the approximating function is closely related to the scenes illumination. The lower the illumination (Lux 3), the smaller the slope of the linear function. This confirms than a change in illumination have an affect on the DN value of the reference panel.

Next the data was analyzed in terms of the affect of the distance between the reference panel and sensor on the DN values. The maximum change in DN values as a function of distance from the reference panel is 500DN, which is merely $1 \%$ of the maximum DN value. This calls for the assumption that during laboratory conditions, the impact of the distance can be neglected.

In order to determine the nomograms, the next step was to determine the dependency between the DN value and the exposure time for each distance at a constant scene illumination. Figure 4 represents this dependence for Lux 1 illuminations for the Master channel.

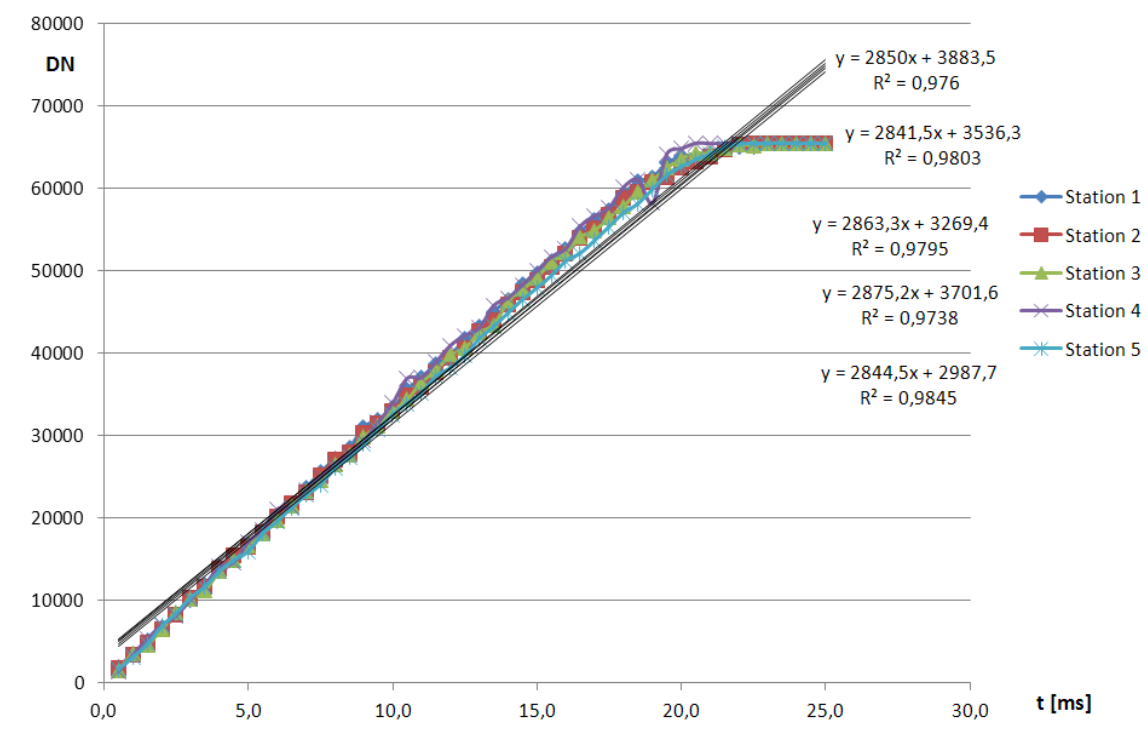

Fig. 4. Dependency between the DN value and the exposure time for each distance at a constant scene illumination 
The above calculations allowed us to construct a nomogram (for each channel separately) for determining the optimal exposure time for a given scene illumination (lux) in order to acquire images, on which the white reference panel would obtain a set percentile value in relation to DNmax. An example nomogram for the Master channel is shown in Figure 5.

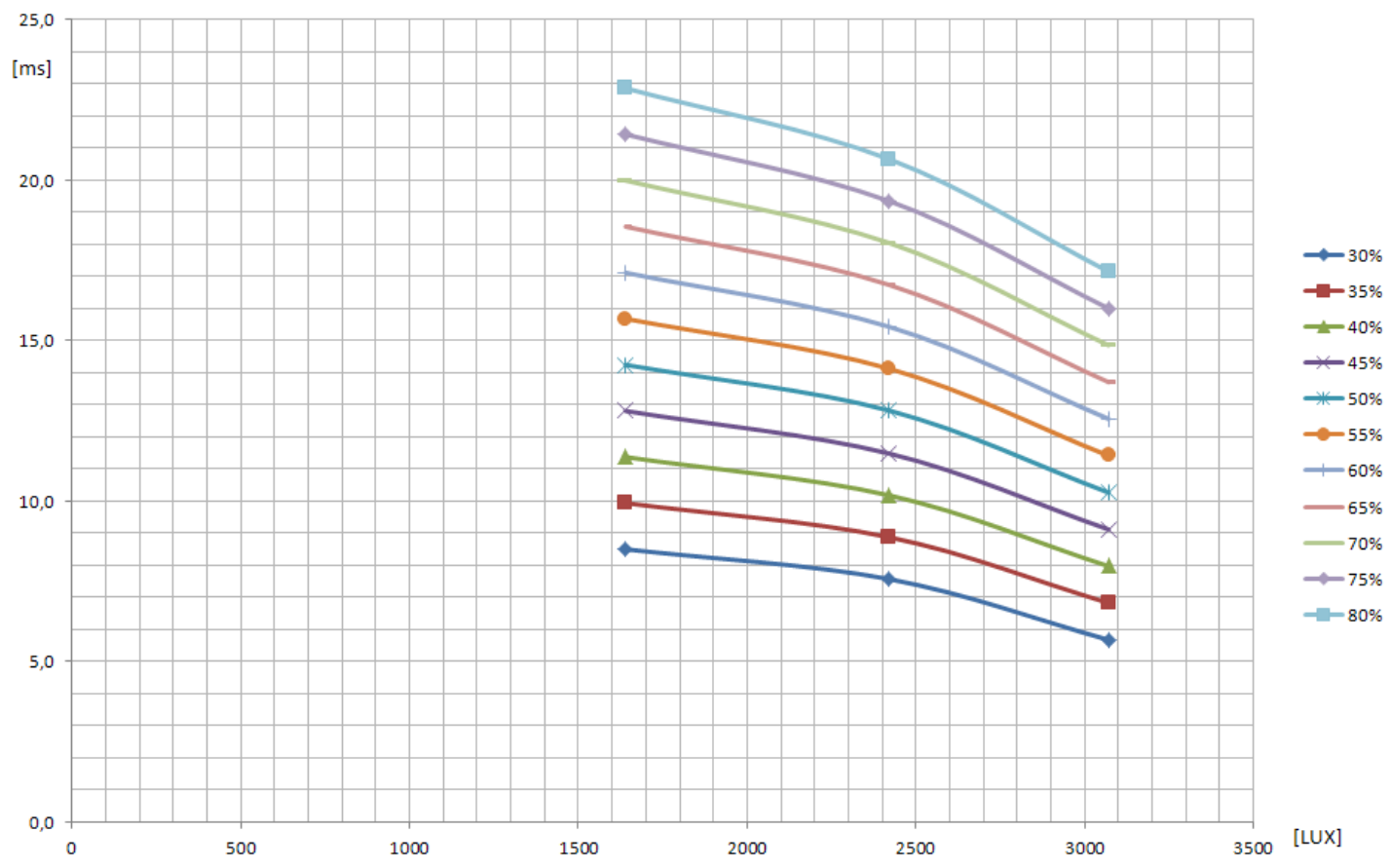

Fig. 5. Master channel nomogram

The obtained nomogram had been derived based on three different illuminations. It is therefore impossible to determine a function which would describe the dependency shown in the figure. However further measurements taken in difference lighting conditions showed that the methodology is correct. The maximum differences between the nomogram data and the new measurements occurred for the Slavel channel and were in the range of $4 \%$ of DNmax.

\section{Summary}

Imagery data acquired with the miniMCA camera have large potential both in terms of their geometry and for obtaining reliable spectral information about studies objects. This translates into the possibility of using it both for typical photogrammetry projects but also in other tasks e.g. precision agriculture, detection of water pollution or asks decision support.

The experiment presented in this article is just the first stage of research, whose aim is to determine a methodology of acquiring reliable spectral information using the mini MCA sensor without the need of using a reference panel within the studied scene. Further experiments will include increasing the accuracy of the presented nomograms and creating a global nomogram for all spectral channels. It is essential to perform additional measurement series in which images will be acquired using at least 4 different illuminations. This would allow us to select the appropriate function to describe this dependency.

The next step would be to mount the camera on a UAV and to perform these experiments in field conditions in order to verify the methodology obtained from laboratory experiments.

\section{Acknowledgements}

The presented article is part of research work carried out in the "Innovative remote sensing system for the monitoring of pollutants in rivers, offshore waters and flooded areas" project - PBS1/B9/8/2012.

\section{References}

[1] Bendig, J.; Bolten, A.; Bareth, G. 2012. Introducing a low-co st mini-UAV For thermal- and multispectral-imaging, International Archives of the Photogrammetry, Remote Sensing and Spatial Information Sciences Volume XXXIX-B1, in 2012 XXII ISPRS Congress, 25 August - 01 September 2012, Melbourne, Australia. 
[2] Kelcey, J.; Lucieer, A. 2012. Sensor correction and radiometric calibration of a 6-band multispectral imaging sensor for uav remote sensing, in International Archives of the Photogrammetry, Remote Sensing and Spatial Information Sciences, Volume XXXIX-BI, 2012 XXII ISPRS Congress, 25 August - 01 September 2012, Melbourne, Australia.

[3] Jose, A. J.; Berni, Student Member, IEEE, Pablo J. Zarco-Tejada; Suárez, L.; Fereres, E. 2009. Thermal and Narrowband Multispectral Remote Sensing for Vegetation Monitoring From an Unmanned Aerial Vehicle, IEEE Transactions on Geoscience and Remote Sensing 47(3).

[4] Tetracam 2011. Tetracam Inc. Digital Camera and Imaging Systems Design - Mini Multiple Camera Array.

[5] Hein, G.; Bento, M. D. F. 2008. Unmanned Aerial Vehicles: An Overview, InsideGNSS 3(1): 54-61.

[6] Laliberte, A.S.; Goforth, M.; Steele, C.; Rango, A. 2011. Multispectral Remote Sensing from Unmanned Aircraft: Image Processing Workflows and Applications for Rangeland Environments, Remote Sens., 3: 2529-2551. http://dx.doi.org/10.3390/rs3112529 